\title{
Generalized Hukuhara Conformable Fractional Derivative and its Application to Fuzzy Fractional Partial DifferentialEquations
}

Manizheh Ghaffari

Islamic Azad University, Science and research branch of Tehran

Tofigh Allahviranloo ( $\nabla$ tofigh.allahviranloo@eng.bau.edu.tr)

Bahcesehir Universitesi https://orcid.org/0000-0002-6673-3560

Saeid Abbasbandy

Imam Khomeini International University

Mahdi Azhini

Islamic Azad university, Science research branch of Tehran

\section{Research Article}

Keywords: Generalized Hukuhara conformable fractional derivative, Fuzzy Traveling wave solution, Generalized partial Hukuhara differentiability, The fuzzy fractional Wave equation, The fuzzy fractional Diffusion equation.

Posted Date: October 18th, 2021

DOI: https://doi.org/10.21203/rs.3.rs-479146/v1

License: (c) (1) This work is licensed under a Creative Commons Attribution 4.0 International License. Read Full License 


\title{
Generalized Hukuhara Conformable Fractional Derivative and its Application to Fuzzy Fractional Partial Differential Equations
}

\author{
Manizheh Ghaffari * Tofigh Allahviranloo * . \\ Saeid Abbasbandy · Mahdi Azhini
}

Received: date / Accepted: date

\begin{abstract}
The main focus of this paper is to develop an efficient analytical method to obtain the traveling wave fuzzy solution for the fuzzy generalized Hukuhara conformable fractional equations by considering the type of generalized Hukuhara conformable fractional differentiability of the solution. To achieve this, the fuzzy conformable fractional derivative based on the generalized Hukuhara differentiability is defined, and several properties are brought on the topic, such as switching points and the fuzzy chain rule. After that, a new analytical method is applied to find the exact solutions for two famous mathematical equations: the fuzzy fractional Wave equation and the fuzzy fractional Diffusion equation. The present work is the first report in which the fuzzy traveling wave method is used to design an analytical method to solve these fuzzy problems. The final examples are asserted that our new method is applicable and efficient.
\end{abstract}

Keywords Generalized Hukuhara conformable fractional derivative · Fuzzy Traveling wave solution - Generalized partial Hukuhara differentiability · The fuzzy fractional Wave equation - The fuzzy fractional Diffusion equation.

Mathematics Subject Classification (2010) 03 E72 $\cdot$ 35L05 $\cdot 35 \mathrm{~K} 05$

Manizheh Ghaffari

Department of Mathematics, Science and Research Branch, Islamic Azad University, Tehran, Iran

Tofigh Allahviranloo

Faculty of engineering and natural sciences, Bahcesehir University, Istanbul, Turkey

Tel.: +902123815717

Fax:+90 2123810550

E-mail: tofigh.allahviranloo@eng.bau.edu.tr

Saeid Abbasbandy

Department of Applied Mathematics, Faculty of Science, Imam Khomeini International University

Mahdi Azhini

Department of Mathematics, Science and Research Branch, Islamic Azad University, Tehran, Iran 


\section{Introduction}

During the last decade, the interest of mathematicians in fuzzy differential equations has been rapidly increasing. The main reason for this development is that using these problems will lead to a much more effective and elegant way of treating real-life issues. A particular subgroup of fuzzy differential equations is described with operators of fractional nature. Fractional calculus is a set of methods and hypotheses that extend the concept of a derivative operator from integer-order $n$ to arbitrary order $\alpha$. Modeling like biological population models, the predator-prey models and infectious diseases models, etc are generalized to fractional order. Fractional calculus is not only a productive and emerging field, but it also represents a new philosophy, how to construct and apply a certain type of nonlocal operator to real-world problems [17, $18,19,26,27,32,33]$.

The interest in fractional fuzzy differential equations aroused in 2012 with a paper by Agarwal et al. [5]. The existence and uniqueness of a fuzzy solution for fractional differential equations are proved in [7]. The concepts of fuzzy fractional integral and Caputo partial differentiability based on generalized Hukuhara differentiability for the fuzzy multivariable functions have been introduced by H. Viet Long et al. [37]. Hoa et al. [20] introduced the fuzzy Caputo-Katugampola fractional differential equations in fuzzy space, and under generalized Lipschitz condition, the existence and uniqueness of the solution proved. The analytical solutions to some linear fractional partial fuzzy differential equations under certain conditions were investigated in [31]. The perturbation-iteration algorithm was developed for numerical solutions of some types of fuzzy fractional partial differential equations with generalized Hukuhara derivative [35]. H. Zureigat et al.[38] analyzed the compact Crank-Nicholson scheme to solve the fuzzy time diffusion equation with fractional order $0<\alpha \leq 1$. Some new methods and useful materials concerning fuzzy fractional differential and fuzzy fractional partial differential equations are introduced in [1].

Recently, a new well-behaved simple fractional derivative called "the conformable fractional derivative" is defined by $[25,6]$. This new definition seems to be a natural extension of the usual derivative. Researchers started to combine this new definition with fuzzy calculus $[22,23,29]$. They used the concept of H-differentiability or strongly generalized differentiability. But it is well-known that the usual Hukuhara difference between two fuzzy numbers exists only under very restrictive conditions $[16,9]$. To overcome this shortcoming, we will introduce the fuzzy conformable fractional derivative under generalized Hukuhara differentiability, and prove some important properties for this kind of differentiability.

Consider the following generic form of second order fuzzy fractional partial differential equation defined based on generalized Hukuhara conformable fractional derivative

$$
\mathscr{D}_{t_{g H}}^{\alpha} v=F\left(v, v_{x_{g H}}, v_{x x_{g H}}, \mathscr{D}_{t_{t_{g}}}^{2 \alpha} v\right)
$$

with $0<\alpha \leq 1$. The main contribution of this paper is to find the wave traveling solutions for the problem (1). For this purpose, the concept of generalized Hukuhara conformable fractional differentiability is introduced thoroughly in the fuzzy functions. 
Next, the fuzzy fractional wave equation and fuzzy Diffusion equation are introduced based on the generalized Hukuhara conformable fractional differentiability. Finally, we discuss the fuzzy traveling wave solutions for these equations by considering the type of $\alpha_{g H}$-differentiability.

We now give a brief outline of the main sections of the paper and state the aims and objectives of each section. Section 3 deals with aspects of background knowledge in fuzzy mathematics and fuzzy derivatives with emphasis on the generalized Hukuhara differentiability. In Section 4, generalized Hukuhara conformable fractional differentiability are studied some properties for this concept of differentiability are proved. A fuzzy fractional Wave equation and a fuzzy fractional diffusion equation under generalized Hukuhara conformable fractional differentiability are introduced in Sections 5 and 6, respectively. Moreover the fuzzy traveling wave solutions of these equations are investigated in different scenarios. Finally in Section 7, the conclusions are given.

\section{Related Works}

The concept of the fuzzy derivative was first introduced by Chang and Zadeh [15]. The starting point of the topic in the set-valued differential equation and also fuzzy differential equation is Hukuhara's paper [21]. Puri and Ralescu [30] suggested the fuzzy differential equations modeling with uncertainty under the concept of $\mathrm{H}$-differentiability. Subsequently, Kaleva in [24] proposed fuzzy differential equations using the Hukuhara derivative, and some other authors developed it. But for some fuzzy differential equations in this framework, the diameter of the solution is unbounded as the time $t$ increases [16].

To overcome this shortcoming, Bede and Gal introduced the weakly generalized differentiability and the strongly generalized differentiability for the fuzzy functions [9]. Moreover, they presented a more general definition of derivatives for the fuzzy functions and their applications for solving fuzzy differential equations [9, 10]. Stefanini and Bede, by the concept of generalization of the Hukuhara difference of compact convex set, introduced generalized Hukuhara differentiability [36] for intervalvalued functions. They showed that this concept of differentiability has relationships with weakly generalized differentiability and strongly generalized differentiability. The disadvantage of the strongly generalized differentiability of a function compared to H-differentiability is that the fuzzy differential equation has no unique solution [9]. Also, in [13] the authors studied relationships between the strongly generalized differentiability and the generalized Hukuhara differentiability, showing the equivalence between these two concepts when the set of switching points of the interval-valued function is finite. 
Table 1 Related Fuzzy Works

\begin{tabular}{|c|c|c|}
\hline Article & Achievements /Advantages & Disadvantages \\
\hline $\begin{array}{l}\text { Chang et al. [15] } \\
\text { (1972) }\end{array}$ & $\begin{array}{l}\text { Define a fuzzy function and its inverse, fuzzy parametric } \\
\text { functions, fuzzy observation, and control. }\end{array}$ & \\
\hline $\begin{array}{l}\text { Hukuhara [21] } \\
(1967)\end{array}$ & $\begin{array}{l}\text { The first definitions of Hukuhara difference, } \\
\text { and Hukuhara derivative }\end{array}$ & $\begin{array}{l}\text { Hukuhara difference between two } \\
\text { fuzzy numbers is not always a fuzzy number. }\end{array}$ \\
\hline $\begin{array}{l}\text { Puri et al.[30] } \\
\text { (1986) }\end{array}$ & $\begin{array}{l}\text { Prove the Ràdström embedding theorem and } \\
\text { define the concept of the differential of a fuzzy function }\end{array}$ & $\begin{array}{l}\text { The diameter of the solution is unbounded } \\
\text { as the time } t \text { increases. }\end{array}$ \\
\hline $\begin{array}{l}\text { Kaleva [24] } \\
(1987)\end{array}$ & $\begin{array}{l}\text { Define a fuzzy differential equations using } \\
\text { the Hukuhara derivative }\end{array}$ & $\begin{array}{l}\text { The diameter of the solution is unbounded } \\
\text { as the time } t \text { increases. }\end{array}$ \\
\hline $\begin{array}{l}\text { Bede et al. [9] } \\
\text { (2005) }\end{array}$ & $\begin{array}{l}\text { Define the strongly generalized differentiability } \\
\text { and the weakly generalized differential of a } \\
\text { fuzzy function using the Hukuhara derivative }\end{array}$ & $\begin{array}{l}\text { The fuzzy differential equations may not } \\
\text { have a unique. }\end{array}$ \\
\hline $\begin{array}{l}\text { Stefanini et al. [36] } \\
\text { (2009) }\end{array}$ & $\begin{array}{l}\text { Define the generalized Hukuhara difference } \\
\text { and generalized Hukuhara differentiability } \\
\text { for interval-valued functions }\end{array}$ & \\
\hline $\begin{array}{l}\text { Allahviranloo et al. [2] } \\
(2015)\end{array}$ & $\begin{array}{l}\text { Define the fuzzy generalized Hukuhara partial } \\
\text { differentiability and solve the fuzzy heat equation }\end{array}$ & \\
\hline $\begin{array}{l}\text { Harir et al. [22] } \\
(2020)\end{array}$ & $\begin{array}{l}\text { Define the fuzzy Generalized Conformable } \\
\text { Fractional Derivative using the Hukuhara derivative }\end{array}$ & $\begin{array}{l}\text { Hukuhara difference between two fuzzy numbers } \\
\text { is not always a fuzzy number. }\end{array}$ \\
\hline $\begin{array}{l}\text { Harir et al. [23] } \\
\text { (2021) }\end{array}$ & $\begin{array}{l}\text { Prove the existence and uniqueness theorem for } \\
\text { a solution to a fuzzy fractional differential equation } \\
\text { by using the concept of conformable differentiability }\end{array}$ & $\begin{array}{l}\text { The diameter of the solution is unbounded } \\
\text { as the time } t \text { increases. }\end{array}$ \\
\hline $\begin{array}{l}\text { Martynyuk et al. [29] } \\
\text { (2020) }\end{array}$ & Define the fractional-like Hukuhara-type derivatives & $\begin{array}{l}\text { Hukuhara difference between two fuzzy numbers } \\
\text { is not always a fuzzy number. }\end{array}$ \\
\hline $\begin{array}{l}\text { Chalco-Cano et al. [14] } \\
(2020)\end{array}$ & $\begin{array}{l}\text { A new characterization of the switching points } \\
\text { for generalized Hukuhara differentiability }\end{array}$ & \\
\hline
\end{tabular}


In this way, they use the LU-parametric representation of fuzzy numbers and fuzzy valued functions to obtain valid approximations of fuzzy generalized Hukuhara derivative and solve fuzzy differential equations [12]. Allahviranloo [2] introduced the fuzzy generalized Hukuhara partial differentiability and solved the fuzzy heat equation under generalized Hukuhara differentiability. Moreover, in [28] the authors obtained the fuzzy solutions of the fuzzy Poisson equation under generalized Hukuhara differentiability. Recently, Y.Chalco-Cano et al. [14] provided a new characterization of the switching points for generalized Hukuhara differentiability and shown that the set of all switching points is at most countable.

\section{Preliminaries}

In the following, we focus on the basic definitions and the necessary notation which will be used throughout the paper. Let $\mathbb{E}$ is the set of fuzzy numbers and $\mathbb{T} \subset \mathbb{E}$ shows the set of all triangular fuzzy numbers.

Let $a=\left(a_{1}, a_{2}, a_{3}\right)$ and $b=\left(b_{1}, b_{2}, b_{3}\right)$ are two triangular fuzzy numbers, so the generalized Hukuhara difference, $a \ominus_{g H} b$, is defined as follows [11]

$$
a \ominus_{g H} b=c \Longleftrightarrow\left\{\begin{array}{l}
(i) \cdot c=\left(a_{1}-b_{1}, a_{2}-b_{2}, a_{3}-b_{3}\right), \\
o r, \quad(i i) . c=\left(a_{3}-b_{3}, a_{2}-b_{2}, a_{1}-b_{1}\right) .
\end{array}\right.
$$

Actually

$$
a \ominus_{g H} b=\left(\min \left\{a_{1}-b_{1}, a_{3}-b_{3}\right\}, a_{2}-b_{2}, \max \left\{a_{1}-b_{1}, a_{3}-b_{3}\right\}\right) .
$$

In this article, we assume that $a \ominus_{g H} b \in \mathbb{T}$.

Let $f:[a, b] \rightarrow \mathbb{T}$ and it's first $k$ generalized Hukuhara derivatives are continuous fuzzy triangular functions without any switching points on domain $\mathbb{I}:=[a, b][11]$.

Definition 31 (See [8]). Let $f: \mathbb{I} \rightarrow \mathbb{E}$ be a fuzzy function and $t_{0} \in \mathbb{I}$. If

$$
\forall \varepsilon>0 \exists \delta>0 \forall t\left(0<\left|t-t_{0}\right|<\delta \Rightarrow D(f(t), L)<\varepsilon\right),
$$

Here, $D$ is the Hausdorff distance. Then, we say that $L \in \mathbb{E}$ is limit of $f$ in $t_{0}$, which is denoted by $\lim _{t \rightarrow t_{0}} f(t)=L$. Also the fuzzy function $f$ is said to be fuzzy continuous if

$$
\lim _{t \rightarrow t_{0}} f(t)=f\left(t_{0}\right)
$$

Theorem 32 (See [3]) Let $f, g: \mathbb{I} \rightarrow \mathbb{E}$ be two fuzzy functions. If $\lim _{t \rightarrow c} f(t)=L_{1}$ and $\lim _{t \rightarrow c} g(t)=L_{2}, L_{1}, L_{2} \in \mathbb{E}$ then

$$
\lim _{t \rightarrow c}\left[f(t) \ominus_{g H} g(t)\right]=L_{1} \ominus_{g H} L_{2} .
$$


Definition 33 (See [11]) The fuzzy function $f(t)$ is generalized Hukuhara differentiable $([\mathrm{gH}]$-differentiable $)$ at $t_{0} \in \mathbb{I}$ if

$$
f_{g H}^{\prime}\left(t_{0}\right)=\lim _{h \rightarrow 0} \frac{f\left(t_{0}+h\right) \Theta_{g H} f\left(t_{0}\right)}{h},
$$

belongs to $\mathbb{E}$. In addition we can say that $f(t)$ is

- $[(i)-g H]$-differentiable function if and only if for all $t \in \mathbb{I}$

$$
f_{i . g H}^{\prime}(t)=\left(f_{1}^{\prime}(t), f_{2}^{\prime}(t), f_{3}^{\prime}(t)\right)
$$

defines a triangular fuzzy number.

- $[(i i)-g H]$-differentiable function if and only if for all $t \in \mathbb{I}$

$$
f_{i i . g H}^{\prime}(t)=\left(f_{3}^{\prime}(t), f_{2}^{\prime}(t), f_{1}^{\prime}(t)\right)
$$

is a triangular fuzzy number.

Proposition 34 (See [34]) Let $\lambda_{1}$ and $\lambda_{2}$ are two real constants such that $\lambda_{1}, \lambda_{2} \geq 0$ (or $\lambda_{1}, \lambda_{2} \leq 0$ ). If $f(t)$ is a triangular fuzzy function, then

$$
\lambda_{1} f(t) \ominus_{g H} \lambda_{2} f(t)=\left(\lambda_{1}-\lambda_{2}\right) f(t) .
$$

Definition 35 (See [1] ]) Let $f: \mathbb{I} \rightarrow \mathbb{T}$ is a fuzzy function and $f(t)=\left(f_{1}(t), f_{2}(t), f_{3}(t)\right)$ and $t_{0} \in \mathbb{I}$ then

$$
\int_{a}^{b} f(t) \mathrm{d} t=\left(\int_{a}^{b} f_{1}(t) \mathrm{d} t, \int_{a}^{b} f_{2}(t) \mathrm{d} t, \int_{a}^{b} f_{3}(t) \mathrm{d} t\right) .
$$

Theorem 36 (See [11]) If $f$ is a gH-differentiable fuzzy function with no switching point in the interval $\mathbb{I}$, then we have

$$
\int_{a}^{b} f_{g H}^{\prime}(t) \mathrm{d} t=f(b) \ominus_{g H} f(a) .
$$

Lemma 37 (See [37]) If $f: \mathbb{I} \rightarrow \mathbb{T}$ be a triangular fuzzy function with no switching point in interval $\mathbb{I}$, then we have

1. If $f(t)$ is $[i-g H]$-differentiable, then

$$
\int_{a}^{b} f_{i . g H}^{\prime}(t) \mathrm{d} t=f(b) \ominus f(a) .
$$

2. If $f(t)$ is $[i i-g H]$-differentiable, then

$$
\int_{a}^{b} f_{i i . g H}^{\prime}(t) \mathrm{d} t=(-1) f(a) \ominus(-1) f(b) .
$$

Lemma 38 (See [28]) $\int_{b}^{a} f(t) \mathrm{d} t=\odot \int_{a}^{b} f(t) \mathrm{d} t$; where $\odot$ denote Hukuhara difference and $f(t)$ be a fuzzy function. 
Definition 39 (See [2]) Let $\left(x_{0}, t_{0}\right) \in \mathbb{D} \subseteq \mathbb{R}^{2}$, then the first generalized Hukuhara partial derivative $([\mathrm{gH}-\mathrm{p}]$-derivative for short) of a fuzzy value function $v(x, t)$ : $\mathbb{D} \rightarrow \mathbb{E}$ at $\left(x_{0}, t_{0}\right)$ with respect to variables $x, t$ are the fuzzy functions $\partial_{x_{g H}} v\left(x_{0}, t_{0}\right)$ and $\partial_{t_{g H}} v\left(x_{0}, t_{0}\right)$ given by

$$
\begin{aligned}
& \partial_{x_{g H}} v\left(x_{0}, t_{0}\right)=\lim _{h \rightarrow 0} \frac{v\left(x_{0}+h, t_{0}\right) \Theta_{g H} v\left(x_{0}, t_{0}\right)}{h}, \\
& \partial_{t_{g H}} v\left(x_{0}, t_{0}\right)=\lim _{k \rightarrow 0} \frac{f\left(x_{0}, t_{0}+k\right) \Theta_{g H} v\left(x_{0}, t_{0}\right)}{k},
\end{aligned}
$$

provided that $\partial_{x_{g H}} v\left(x_{0}, t_{0}\right)$ and $\partial_{t_{g H}} v\left(x_{0}, t_{0}\right) \in \mathbb{E}$.

Definition 310 (See [2]) A triangular fuzzy function $v(x, t)=\left(v_{1}(x, t), v_{2}(x, t), v_{3}(x, t)\right)$, without any switching points on $\mathbb{D}$ is called

- $[(i)-p]$-differentiable with respect to $t$ at $\left(x_{0}, t_{0}\right)$ if and only if

$$
v_{t_{i . g H}}\left(x_{0}, t_{0}\right)=\left.\left(\frac{\partial v_{1}(x, t)}{\partial t}, \frac{\partial v_{2}(x, t)}{\partial t}, \frac{\partial v_{3}(x, t)}{\partial t}\right)\right|_{x=x_{0}, t=t_{0}}
$$

defines a triangular fuzzy number, and

- it's $[(i i)-p]$-differentiable if and only if

$$
v_{t_{i i . g H}}\left(x_{0}, t_{0}\right)=\left.\left(\frac{\partial v_{3}(x, t)}{\partial t}, \frac{\partial v_{2}(x, t)}{\partial t}, \frac{\partial v_{1}(x, t)}{\partial t}\right)\right|_{x=x_{0}, t=t_{0}},
$$

defines a triangular fuzzy number.

Moreover, if $v_{x}(x, t)$ is $[g H-p]$-differentiable at $(x, t)$ with respect to $x$ without any switching point on $\mathbb{D}$ and

- if the type of $[g H-p]$-differentiability of both $v(x, t)$ and $v_{x}(x, t)$ are the same, then $v_{x}(x, t)$ is $[(i)-p]$-differentiable w.r.t $x$ and

$$
v_{x x_{i . g H}}\left(x_{0}, t_{0}\right)=\left.\left(\frac{\partial^{2} v_{1}(x, t)}{\partial x^{2}}, \frac{\partial^{2} v_{2}(x, t)}{\partial x^{2}}, \frac{\partial^{2} v_{3}(x, t)}{\partial x^{2}}\right)\right|_{x=x_{0}, t=t_{0}}
$$

- if the type of $[g H-p]$-differentiability $v(x, t)$ and $v_{x}(x, t)$ are different, therefore $v_{x}(x, t)$ is $[(i i)-p]$-differentiable w.r.t $x$ and

$$
v_{x x_{i i . g H}}\left(x_{0}, t_{0}\right)=\left.\left(\frac{\partial^{2} v_{3}(x, t)}{\partial x^{2}}, \frac{\partial^{2} v_{2}(x, t)}{\partial x^{2}}, \frac{\partial^{2} v_{1}(x, t)}{\partial x^{2}}\right)\right|_{x=x_{0}, t=t_{0}}
$$




\section{Generalized Hukuhara Conformable Fractional Derivative}

In this section, we are going to introduce conformable fractional derivative based on the generalized Hukuhara derivative. Moreover, we will prove several properties for this kind of differentiability.

Definition 41 Let $f:[0, \infty) \rightarrow \mathbb{E}$ be a triangular fuzzy function. The generalized Hukuhara conformable fractional derivative of $f$ of order $\alpha \in(0,1)$ is defined by

$$
T_{g H}^{\alpha}(f)(t)=\lim _{\varepsilon \rightarrow 0} \frac{f\left(t+\varepsilon t^{1-\alpha}\right) \ominus_{g H} f(t)}{\varepsilon},
$$

provided that $T_{g H}^{\alpha}(f)(t) \in \mathbb{E}$. If the generalized Hukuhara conformable fractional derivative of $f$ of order $\alpha$ exists, then we simply say $f$ is $\alpha_{g H}$-differentiable.

Theorem 42 If a fuzzy function $f:[0, \infty) \rightarrow \mathbb{E}$ is $\alpha_{g H}$-differentiable at $t_{0}>0, \alpha \in$ $(0,1]$, then $f$ is continuous at $t_{0}$.

Proof We have $f\left(t+\varepsilon t^{1-\alpha}\right) \ominus_{g H} f(t)=\frac{f\left(t+\varepsilon t^{1-\alpha}\right) \ominus_{g H} f(t)}{\varepsilon} \odot \varepsilon$. By using Theorem 32, we conclude that

$$
\lim _{\varepsilon \rightarrow 0}\left[f\left(t+\varepsilon t^{1-\alpha}\right) \ominus_{g H} f(t)\right]=\lim _{\varepsilon \longrightarrow 0} \frac{f\left(t+\varepsilon t^{1-\alpha}\right) \ominus_{g H} f(t)}{\varepsilon} \odot \lim _{\varepsilon \longrightarrow 0} \varepsilon
$$

then

$$
\lim _{\varepsilon \rightarrow 0}\left[f\left(t+\varepsilon t^{1-\alpha}\right) \ominus_{g H} f(t)\right]=T_{g H}^{\alpha}(f)(t) \odot 0 .
$$

Now, let $h=\varepsilon t_{0}^{1-\alpha}$, therefore

$$
\lim _{h \rightarrow 0}\left[f(t+h) \ominus_{g H} f(t)\right]=0 .
$$

Therefore, according to Definition 31, it can be concluded that the function $f$ is fuzzy continuous.

Definition 43 Let $f:[0, \infty) \rightarrow \mathbb{E}$ be a triangular fuzzy function. The generalized Hukuhara conformable fractional derivative of $f$ of order $\beta \in(1,2)$ is defined by

$$
T_{g H}^{\beta}(f)(t)=\lim _{\varepsilon \rightarrow 0} \frac{f_{g H}^{\prime}\left(t+\varepsilon t^{2-\beta}\right) \ominus_{g H} f_{g H}^{\prime}(t)}{\varepsilon},
$$

provided that $T_{g H}^{\beta}(f)(t) \in \mathbb{E}$. If the generalized Hukuhara conformable fractional derivative of $f$ of order $\beta$ exists, then we simply say $f$ is $\beta_{g H}$-differentiable.

Definition 44 Let $\alpha \in(0,1)$ and $f$ is $\alpha_{g H}$-differentiable at a point $t>0$. We can say that $f(t)$ is

$-\alpha_{i . g H}$-differentiable function if and only if for all $t>0$

$$
T_{i . g H}^{\alpha}(f)(t)=\left(T^{\alpha}\left(f_{1}\right)(t), T^{\alpha}\left(f_{2}\right)(t), T^{\alpha}\left(f_{3}\right)(t)\right),
$$

defines a triangular fuzzy number. 


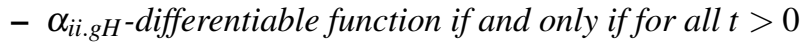

$$
T_{i i . g H}^{\alpha}(f)(t)=\left(T^{\alpha}\left(f_{3}\right)(t), T^{\alpha}\left(f_{2}\right)(t), T^{\alpha}\left(f_{1}\right)(t)\right),
$$

be a triangular fuzzy number.

Here, $T^{\alpha}\left(f_{i}\right)(t), i=1,2,3$ is the conformable fractional derivative for the real valued function $f_{i}(t)[25]$.

Definition 45 We say that a point $\xi_{0} \in(0, \infty)$, is a switching point for the differentiability of $f$, if in any neighborhood $\mathscr{V}$ of $\xi_{0}$ there exist points $\xi_{1}<\xi_{0}<\xi_{2}$ such that

Type I. at $\xi_{1}$ (5) holds while (6) does not hold and at $\xi_{2}$ (6) holds and (5) does not hold, or

Type II. at $\xi_{1}(6)$ holds while (5) does not hold and at $\xi_{2}(5)$ holds and (6) does not hold.

Theorem 46 Let $\alpha \in(0,1)$ and $f$ be $\alpha_{g H}$-differentiable at a point $t>0$. Then

$$
T_{g H}^{\alpha}(f)(t)=t^{1-\alpha} f_{g H}^{\prime}(t) .
$$

Proof In Definition 41, let $h=\varepsilon t^{1-\alpha}$ and then $\varepsilon=t^{\alpha-1} h$. Hence

$$
\begin{aligned}
T_{g H}^{\alpha}(f)(t) & =\lim _{\varepsilon \rightarrow 0} \frac{f\left(t+\varepsilon t^{1-\alpha}\right) \ominus_{g H} f(t)}{\varepsilon} \\
& =\lim _{h \rightarrow 0} \frac{f(t+h) \ominus_{g H} f(t)}{h t^{\alpha-1}} \\
& =t^{1-\alpha} \lim _{h \rightarrow 0} \frac{f(t+h) \ominus_{g H} f(t)}{h} \\
& =t^{1-\alpha} f_{g H}^{\prime}(t) .
\end{aligned}
$$

So the desired result was obtained.

Remark 47 Using Theorem 46 and Definition 45 , it can be similarly easily shown that for $\beta \in(1,2)$

$$
T_{g H}^{\beta}(f)(t)=t^{2-\beta} f_{g H}^{\prime \prime}(t)
$$

where $f$ is $g H$-differentiable of second order.

Example 48 Consider the fuzzy function $f:[0, \pi] \rightarrow \mathbb{E}$ defined by

$$
f(t)=(1.3 \sin (t), 5.2 \sin (t), 9.6 \sin (t)) .
$$

We have the following $\alpha_{g H}$-derivatives of $f(t)$

$$
\left\{\begin{array}{l}
T_{g H}^{\frac{1}{2}}(f)(t)=\left(1.3 t^{\frac{1}{2}} \cos (t), 5.2 t^{\frac{1}{2}} \cos (t), 9.6 t^{\frac{1}{2}} \cos (t)\right) t \in\left[0, \frac{\pi}{2}\right], \\
T_{g H}^{\frac{1}{2}}(f)(t)=\left(9.6 t^{\frac{1}{2}} \cos (t), 5.2 t^{\frac{1}{2}} \cos (t), 1.3 t^{\frac{1}{2}} \cos (t)\right) t \in\left[\frac{\pi}{2}, \pi\right] .
\end{array}\right.
$$



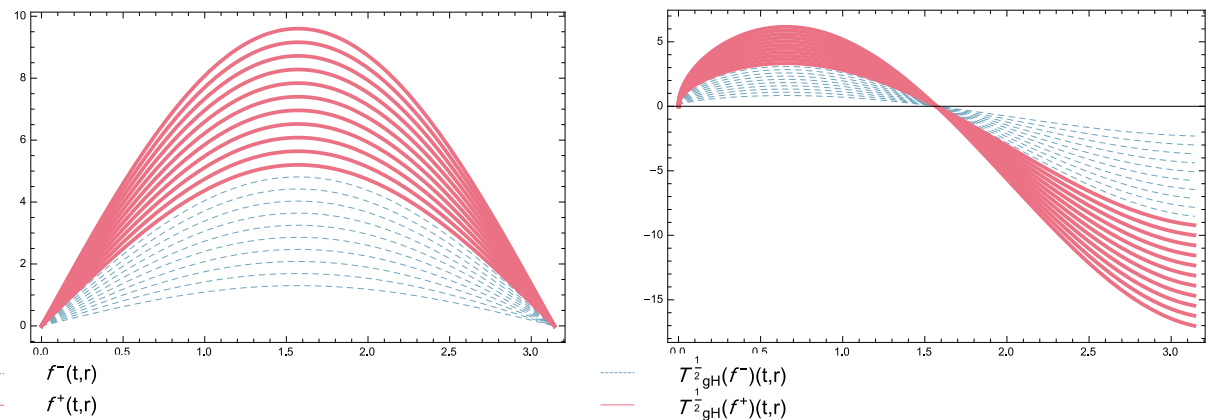

Fig. $1 r$-cut representation of $f(t)$ (left) and its $T_{g H}^{\frac{1}{2}}(f)(t)$ (right) for $r \in[0,1]$ of Example 48

Therefore, the fuzzy function $f(t)$ is $\alpha_{i . g H}$-differentiable function on $t \in\left[0, \frac{\pi}{2}\right]$. This function is switched to $\alpha_{i i . g H}$-differentiable at $t=\frac{\pi}{2}$. Hence, the point $t=\frac{\pi}{2}$ is a switching point of Type I for the the differentiability of $f$.

Theorem 49 Let $g: \mathbb{I} \rightarrow \zeta$ is real valued differentiable at $t$, and $f: \zeta \rightarrow \mathbb{E}$ be a fuzzy function such that $f$ is $g H$-differentiable at the point $g(t)$ without any switching points, and $\alpha \in(0,1)$.

- Assume $f(t)$ is $[(i)-g H]$-differentiable at $g(t)$, then function $(f o g)(t)$ is $\alpha_{i . g H^{-}}$ differentiable if

$$
T_{i . g H}^{\alpha}(f \circ g)(t)= \begin{cases}t^{1-\alpha} g^{\prime}(t) \odot f_{i . g H}^{\prime}(g(t)), & \text { If } g^{\prime}(t)>0, \\ \ominus(-1) t^{1-\alpha} g^{\prime}(t) \odot f_{i . g H}^{\prime}(g(t)), \text { If } g^{\prime}(t)<0 .\end{cases}
$$

- Let $f(t)$ is $[(i i)-g H]$-differentiable at $g(t)$, then the function $(f o g)(t)$ is $\alpha_{i i . g H^{-}}$ differentiable if

$$
T_{i i . g H}^{\alpha}(f \circ g)(t)= \begin{cases}t^{1-\alpha} g^{\prime}(t) \odot f_{i i . g H}^{\prime}(g(t)), & \text { If } g^{\prime}(t)>0, \\ \ominus(-1) t^{1-\alpha} g^{\prime}(x) \odot f_{i i . g H}^{\prime}(g(x)), \text { If } g^{\prime}(t)<0 .\end{cases}
$$

Proof First let $f(t)$ is $[(i)-g H]$-differentiable at $g(t)$. We have the following cases

i. If $g^{\prime}(t)>0$. Hence by attention to Theorem 46 we have

$$
\begin{aligned}
t^{1-\alpha} g^{\prime}(t) & \odot\left(f_{1}^{\prime}(g(t)), f_{2}^{\prime}(g(t)), f_{3}^{\prime}(g(t))\right) \\
= & \left(t^{1-\alpha} g^{\prime}(t) f_{1}^{\prime}(g(t)), t^{1-\alpha} g^{\prime}(t) f_{2}^{\prime}(g(t)), t^{1-\alpha} g^{\prime}(t) f_{3}^{\prime}(g(t))\right) \\
= & T_{i . g H}^{\alpha}(f o g)(t) .
\end{aligned}
$$


ii. If $g^{\prime}(t)<0$, then

$$
\begin{aligned}
\ominus(-1) t^{1-\alpha} g^{\prime}(t) \odot\left(f_{1}^{\prime}(g(t)), f_{2}^{\prime}(g(t)), f_{3}^{\prime}(g(t))\right) \\
=\ominus(-1)\left(t^{1-\alpha} g^{\prime}(t) f_{3}^{\prime}(g(t)), t^{1-\alpha} g^{\prime}(t) f_{2}^{\prime}(g(t)), t^{1-\alpha} g^{\prime}(t) f_{1}^{\prime}(g(t))\right) \\
=\ominus\left(-t^{1-\alpha} g^{\prime}(t) f_{1}^{\prime}(g(t)),-t^{1-\alpha} g^{\prime}(x) f_{2}^{\prime}(g(t)),-t^{1-\alpha} g^{\prime}(t) f_{3}^{\prime}(g(t))\right) \\
=\left(t^{1-\alpha} g^{\prime}(t) f_{1}^{\prime}(g(t)), t^{1-\alpha} g^{\prime}(t) f_{2}^{\prime}(g(t)), t^{1-\alpha} g^{\prime}(t) f_{3}^{\prime}(g(t))\right) \\
=T_{i . g H}^{\alpha}(f o g)(t) .
\end{aligned}
$$

We can use the same procedure when $f(t)$ is $[(i i)-g H]$-differentiable at $g(t)$.

\section{Fuzzy Traveling Wave Solution of The Fuzzy Fractional Wave Equation}

We want to find traveling wave fuzzy solution of the fuzzy one-dimensional homogeneous fractional wave equation. Consider this problem as follows

$$
\left\{\begin{array}{l}
\mathscr{D}_{t t_{g H}}^{2 \alpha} v \ominus_{g H} \kappa^{2} \odot v_{x x_{g H}}=0, \quad(x, t) \in \mathbb{R} \times[0, \infty), \\
v(x, 0)=f(x), \quad \mathscr{D}_{t_{g H}}^{\alpha} v(x, 0)=g(x)
\end{array}\right.
$$

Where $\alpha \in\left(\frac{1}{2}, 1\right)$ and $\mathscr{D}_{t_{g H}}^{\alpha}$ is the generalized Huhuhara conformable fractional partial derivatives with respect to $t$ and $v_{x_{g H}}$ is the generalized Hukuhara partial derivative with respect to $x$. Here, $f(x), g(x)$ are given continuous fuzzy functions. We will find the triangular analytical fuzzy solutions of Eq.(7) by using traveling wave method provided that the types of $\alpha_{g H}$-differentiability of $v(x, t)$ with respect to $t$ and $[g H-$ $p$ ]-differentiability with respect to $x$ are the same. By considering the type of $\alpha_{g H^{-}}$ differentiability of $v(x, t)$ with respect to $t$, we have different cases as follow:

1-1-gH . Let $v(x, t)$ and $\mathscr{D}_{t_{g H}}^{\alpha} v$ are $\alpha_{i . g H}$-differentiable with respect to $t$, and $v(x, t)$ and $v_{x_{g H}}$ are $[i-p]$-differentiable with respect to $x$. Then $\mathscr{U}(\xi)$ is a $[(i)-g H]$ differentiable fuzzy. Here, we outline the main steps of traveling wave method. function

Step 1. Consider the fuzzy one-dimensional homogeneous fractional wave equation (7)

$$
v(x, t)=\mathscr{U}(\xi), \quad \text { where } \quad \xi=x-\gamma^{t^{\alpha}}
$$

which can be analyzed through a change of variables $v(x, t)=\mathscr{U}(\xi)$. Here, $\mathscr{U}$ is a continuous function and $g H$-differentiable in $\xi$ and $\gamma$ is a positive real constant.

Step 2. We have

$$
\frac{\partial \xi}{\partial t}=-\gamma t^{\alpha-1}<0, \quad \frac{\partial \xi}{\partial x}=1>0
$$


therefore, by using the Theorem 49, the fuzzy multivariate chain rule [28], we have

$$
\begin{aligned}
\mathscr{D}_{t i g H}^{\alpha} v & =t^{1-\alpha} \odot \frac{d_{i . g H} \mathscr{U}}{d \xi} \odot \frac{\partial \xi}{\partial t}=\ominus \gamma \odot \frac{d_{i . g H} \mathscr{U}}{d \xi}, \Rightarrow \mathscr{D}_{t t_{i . g H}}^{2 \alpha} v=\gamma^{2} \odot \frac{d_{i . g H}^{2} \mathscr{U}}{d \xi^{2}}, \\
v_{x_{i . g H}} & =\frac{d_{i . g H} \mathscr{U}}{d \xi} \odot \frac{\partial \xi}{\partial x}=\frac{d_{i . g H} \mathscr{U}}{d \xi}, \Rightarrow \quad v_{x x_{i . g H}}=\frac{d_{i . g H}^{2} \mathscr{U}}{d \xi^{2}} .
\end{aligned}
$$

Hence the equation (7) is reduced to the following fuzzy ordinary differential equations of $\xi$

$$
\gamma^{2} \frac{d_{i . g H}^{2} \mathscr{U}}{d \xi^{2}} \Theta_{g H} \kappa^{2} \frac{d_{i . g H}^{2} \mathscr{U}}{d \xi^{2}}=0 .
$$

Step 3. To find fuzzy solutions for ordinary differential equations (8) and (17), we need some auxiliary boundary conditions. Which in this article, we consider the following auxiliary boundary conditions

$$
\lim _{\xi \rightarrow \pm \infty} \mathscr{U}(\xi)=0, \quad \lim _{\xi \rightarrow \pm \infty} \frac{d \mathscr{U}}{d \xi}=0, \quad \lim _{\xi \rightarrow \pm \infty} \frac{d^{2} \mathscr{U}}{d \xi^{2}}=0 .
$$

By using Proposition 34, Eq.(17) can also be written as follows

$$
\left(\gamma^{2}-\kappa^{2}\right) \frac{d_{i . g H}^{2} \mathscr{U}}{d \xi^{2}}=0
$$

One possibility is for $\frac{d_{i . g H}^{2} \mathscr{U}}{d \xi^{2}}=0$. In which case we have

$$
\mathscr{U}(\xi)=\mathscr{C}_{1} \oplus \mathscr{C}_{2} \xi \Rightarrow v(x, t)=\mathscr{C}_{1} \oplus \mathscr{C}_{2}\left(x-\gamma \frac{t^{\alpha}}{\alpha}\right)
$$

where $\mathscr{C}_{1}$ and $\mathscr{C}_{2}$ are fuzzy integral constants. But the boundary conditions (9) cannot be satisfied unless $\mathscr{C}_{2}=0$. Thus the only traveling solution is a fuzzy constant.

Another possibility is for $\gamma^{2}=\kappa^{2}$. In this case

$$
v(x, t)=\mathscr{U}\left(x-\kappa \frac{t^{\alpha}}{\alpha}\right), \quad v(x, t)=\mathscr{U}\left(x+\kappa \frac{t^{\alpha}}{\alpha}\right)
$$

are traveling wave solutions of thefuzzy fractional wave equation and $\mathscr{U}$ can be any two $g H$-differentiable function. In general, it follows that any solution to the fuzzy fractional wave equation can be obtained as a superposition of two traveling waves,

$$
v(x, t)=\mathscr{F}\left(x+\kappa \frac{t^{\alpha}}{\alpha}\right) \oplus \mathscr{G}\left(x-\kappa \frac{t^{\alpha}}{\alpha}\right)
$$

Since equation (12) is a fuzzy solution for equation (7), then it must apply to the initial conditions of the equation (7)

$$
v(x, 0)=f(x), \quad D_{t_{g H}}^{\alpha} v(x, 0)=g(x) .
$$


Hence, the initial condition $v(x, 0)=f(x)$ concludes

$$
\mathscr{F}(x) \oplus \mathscr{G}(x)=f(x) .
$$

By considering Theorem 49 we have

$$
D_{t_{g H}}^{\alpha} v(x, t)=\kappa \odot \mathscr{F}_{i . g H}^{\prime}\left(x+\kappa \frac{t^{\alpha}}{\alpha}\right) \ominus \kappa \odot \mathscr{G}_{i . g H}^{\prime}\left(x-\kappa \frac{t^{\alpha}}{\alpha}\right),
$$

By the initial condition $D_{t_{g H}}^{\alpha} v(x, 0)=g(x)$, we can write

$$
\mathscr{F}_{i . g H}^{\prime}(x) \ominus \mathscr{G}_{i . g H}^{\prime}(x)=\frac{1}{\kappa} g(x)
$$

After integration by using Lemma 37

$$
(\mathscr{F}(x) \ominus \mathscr{F}(0)) \ominus(\mathscr{G}(x) \ominus \mathscr{G}(0))=\frac{1}{\kappa} \int_{0}^{x} g(s) d s \Rightarrow \mathscr{F}(x) \ominus \mathscr{G}(x)=(\mathscr{F}(0) \ominus \mathscr{G}(0)) \oplus \frac{1}{\kappa} \int_{0}^{x} g(s) d s
$$

The following system of equations is obtained by Eqs.(19) and (15)

$$
\left\{\begin{array}{l}
\mathscr{F}(x) \oplus \mathscr{G}(x)=f(x), \\
\mathscr{F}(x) \ominus \mathscr{G}(x)=(\mathscr{F}(0) \ominus \mathscr{G}(0)) \oplus \frac{1}{\kappa} \int_{0}^{x} g(s) d s,
\end{array}\right.
$$

such that this system of equations has the following fuzzy solutions

$$
\begin{gathered}
\mathscr{F}(x)=\frac{1}{2} f(x) \oplus \frac{1}{2}(\mathscr{F}(0) \ominus \mathscr{G}(0)) \oplus \frac{1}{2 \kappa} \int_{0}^{x} g(s) d s, \\
\mathscr{G}(x)=\frac{1}{2} f(x) \ominus \frac{1}{2}(\mathscr{F}(0) \ominus \mathscr{G}(0)) \ominus \frac{1}{2 \kappa} \int_{0}^{x} g(s) d s,
\end{gathered}
$$

On the other hand, according to Lemma $38, \mathscr{G}(x)$ can be rewritten as follows

$$
\mathscr{G}(x)=\frac{1}{2} f(x) \ominus \frac{1}{2}(\mathscr{F}(0) \ominus \mathscr{G}(0)) \oplus \frac{1}{2 \kappa} \int_{x}^{0} g(s) d s
$$

By substituting these equations for $\mathscr{F}$ and $\mathscr{G}$ into the general solution (12), the fuzzy traveling wave solution is obtained as follow

$$
v(x, t)=\frac{1}{2}\left(f\left(x+\kappa \frac{t^{\alpha}}{\alpha}\right) \oplus f\left(x-\kappa \frac{t^{\alpha}}{\alpha}\right)\right) \oplus \frac{1}{2 \kappa} \int_{x-\kappa \frac{t}{\alpha}}^{x+\kappa \frac{t^{\alpha}}{\alpha}} g(s) d s
$$

Here, $v(x, t)$ and $\mathscr{D}_{t_{g H}}^{\alpha} v$ are $\alpha_{i . g H}$-differentiable with respect to $t$, and $v(x, t)$ and $v_{x_{g H}}$ are $[(i)-g H]$-differentiable with respect to $x$.

2-2-gH . Let $v(x, t)$ and $\mathscr{D}_{t_{g H}}^{\alpha} v$ are $\alpha_{i i . g H}$-differentiable with respect to $t$ and $v(x, t)$ and $v_{x_{g H}}$ are $[(i i)-g H]$-differentiable with respect to $x$ then $\mathscr{U}(\xi)$ is a $[(i i)-g H]$ differentiable fuzzy function. In this case, the main steps of the fuzzy traveling wave method are as follows 
Step 1. Let we can analyzed he fuzzy one-dimensional homogeneous fractional wave equation (7) through the following change variables

$$
v(x, t)=\mathscr{U}(\xi), \quad \text { where } \quad \xi=x-\gamma \frac{t^{\alpha}}{\alpha},
$$

where $\mathscr{U}$ is a continuous function and $g H$-differentiable in $\xi$ and $\gamma$ is a positive real constant.

Step 2. We have

$$
\frac{\partial \xi}{\partial t}=-\gamma t^{\alpha-1}<0, \quad \frac{\partial \xi}{\partial x}=1>0,
$$

therefore, by using the Theorem 49, the fuzzy multivariate chain rule [28], we have

$$
\begin{aligned}
\mathscr{D}_{\text {ii. } g H}^{\alpha} v & =t^{1-\alpha} \odot \frac{d_{i i . g H} \mathscr{U}}{d \xi} \odot \frac{\partial \xi}{\partial t}=\ominus \gamma \odot \frac{d_{i i . g H} \mathscr{U}}{d \xi}, \Rightarrow \mathscr{D}_{t t_{i i . g H}}^{2 \alpha} v=\gamma^{2} \odot \frac{d_{i i . g H}^{2} \mathscr{U}}{d \xi^{2}} . \\
v_{x_{i i . g H}} & =\frac{d_{i i . g H} \mathscr{U}}{d \xi} \odot \frac{\partial \xi}{\partial x}=\frac{d_{i i . g H} \mathscr{U}}{d \xi}, \Rightarrow v_{x x_{i i . g H}}=\frac{d_{i i . g H}^{2} \mathscr{U}}{d \xi^{2}} .
\end{aligned}
$$

Hence the equation (7) is reduced to the following fuzzy ordinary differential equations of $\xi$

$$
\gamma^{2} \frac{d_{i i . g H}^{2} \mathscr{U}}{d \xi^{2}} \ominus_{g H} \kappa^{2} \frac{d_{i i . g H}^{2} \mathscr{U}}{d \xi^{2}}=0 .
$$

Step 3. As we explained in case 1-1-gH, any solution of the fuzzy fractional wave equation can be obtained as follow

$$
v(x, t)=\mathscr{F}\left(x+\kappa \frac{t^{\alpha}}{\alpha}\right) \oplus \mathscr{G}\left(x-\kappa \frac{t^{\alpha}}{\alpha}\right) .
$$

Equation (18) is a fuzzy solution for equation (7), then the initial condition $v(x, 0)=f(x)$ yields

$$
\mathscr{F}(x) \oplus \mathscr{G}(x)=f(x) .
$$

On the other hand, using Theorem 49 and the initial value $D_{t_{g H}}^{\alpha} v(x, 0)=g(x)$, we have

$v_{t_{i i . g H}}(x, t)=\kappa \odot \mathscr{F}_{i i . g H}^{\prime}\left(x+\kappa \frac{t^{\alpha}}{\alpha}\right) \ominus \kappa \odot \mathscr{G}_{i i . g H}^{\prime}\left(x-\kappa \frac{t^{\alpha}}{\alpha}\right), \quad \Rightarrow \quad \kappa \mathscr{F}_{i i . g H}^{\prime}(x) \ominus \kappa \mathscr{G}_{i i . g H}^{\prime}(x)=g(x)$.

Integrate each side of the above equation by using Lemma 37, therefore

$$
((-1) \mathscr{F}(0) \ominus(-1) \mathscr{F}(x)) \ominus((-1) \mathscr{G}(0) \ominus(-1) \mathscr{G}(x))=\frac{1}{\kappa} \int_{0}^{x} g(s) d s,
$$

and

$$
\mathscr{G}(x) \ominus \mathscr{F}(x)=(\mathscr{G}(0) \ominus \mathscr{F}(0)) \oplus \frac{(-1)}{\kappa} \int_{0}^{x} g(s) d s .
$$


Consequently, we find that

$$
\left\{\begin{array}{l}
\mathscr{F}(x) \oplus \mathscr{G}(x)=f(x), \\
\mathscr{G}(x) \ominus \mathscr{F}(x)=(\mathscr{G}(0) \ominus \mathscr{F}(0)) \oplus \frac{(-1)}{\kappa} \int_{0}^{x} g(s) d s .
\end{array}\right.
$$

By solving this system and using Lemma 38, the following results are obtained

$$
\begin{aligned}
\mathscr{G}(x) & =\frac{1}{2} f(x) \oplus \frac{1}{2}(\mathscr{G}(0) \ominus \mathscr{F}(0)) \ominus \frac{(-1)}{2 \kappa} \int_{x}^{0} g(s) d s, \\
\mathscr{F}(x) & =\frac{1}{2} f(x) \ominus \frac{1}{2}(\mathscr{G}(0) \ominus \mathscr{F}(0)) \ominus \frac{(-1)}{2 \kappa} \int_{0}^{x} g(s) d s .
\end{aligned}
$$

So the final solution of Eq. (7) is

$$
v(x, t)=\frac{1}{2}\left(f\left(x+\kappa \frac{t^{\alpha}}{\alpha}\right) \oplus f\left(x-\kappa \frac{t^{\alpha}}{\alpha}\right)\right) \ominus \frac{(-1)}{2 \kappa} \int_{x-\kappa \frac{t^{\alpha}}{\alpha}}^{x+\kappa \frac{t^{\alpha}}{\alpha}} g(s) d s .
$$

Where $v(x, t)$ and $\mathscr{D}_{t_{g H}}^{\alpha} v$ are $\alpha_{i i . g H}$-differentiable with respect to $t$, and $v(x, t)$ and $v_{x_{g H}}$ are $[(i i)-g H]$-differentiable with respect to $x$.

Example 51 Consider the following fuzzy fractional wave equation

$$
\begin{cases}\mathscr{D}_{t_{g} H}^{\frac{7}{4}} v \ominus_{g H} v_{x x_{g H}}=0, & (x, t) \in \mathbb{R} \times(0, \infty), \\ v(x, 0)=(3.9 x, 6.7 x, 9.5 x), & \mathscr{D}_{t_{g H}}^{\frac{7}{8}} v(x, 0)=(3.9,6.7,9.5) .\end{cases}
$$

To find a $1-1-g H$-differentiable solution for this problem, we use the equation (16)

$$
\begin{aligned}
v(x, t)= & \frac{1}{2}\left(f\left(x+\kappa \frac{t^{\alpha}}{\alpha}\right) \oplus f\left(x-\kappa \frac{t^{\alpha}}{\alpha}\right)\right) \oplus \frac{1}{2 \kappa} \int_{x-\kappa \frac{t^{\alpha}}{\alpha}}^{x+\kappa t^{\alpha}} g(s) d s \\
= & \left(\frac{3.9}{2}\left(\left(x+\frac{8}{7} t^{\frac{7}{8}}\right)+\left(x-\frac{8}{7} t^{\frac{7}{8}}\right)\right), \frac{6.7}{2}\left(\left(x+\frac{8}{7} t^{\frac{7}{8}}\right)+\left(x-\frac{8}{7} t^{\frac{7}{8}}\right)\right), \frac{9.5}{2}\left(\left(x+\frac{8}{7} t^{\frac{7}{8}}\right)+\left(x-\frac{8}{7} t^{\frac{7}{8}}\right)\right)\right) \\
& \oplus\left(\frac{1}{2} \int_{x-\frac{8}{7} t^{\frac{7}{8}}}^{x+\frac{8}{7} t^{\frac{7}{8}}} 3.9 \mathrm{~d} s, \frac{1}{2} \int_{x-\frac{8}{7} t^{\frac{7}{8}}}^{x+\frac{8}{7} t^{\frac{7}{8}}} 6.7 \mathrm{~d} s, \frac{1}{2} \int_{x-\frac{8}{7} t^{\frac{7}{8}}}^{x+\frac{8}{7} t^{\frac{7}{8}}} 9.5 \mathrm{~d} s\right) \\
= & \left(\frac{31.2}{7} t^{\frac{7}{8}}+3.9 x, \frac{53.6}{7} t^{\frac{7}{8}}+6.7 x, \frac{76}{7} t^{\frac{7}{8}}+9.5 x\right)
\end{aligned}
$$

Example 52 Consider the following fuzzy fractional wave equation

$$
\left\{\begin{array}{l}
\mathscr{D}_{t_{t_{g}}^{\frac{3}{2}} v \ominus_{g H} v_{x x_{g H}}=0,} \quad(x, t) \in \mathbb{R} \times(0, \infty), \\
v(x, 0)=\left(2.1 x^{2}, 5 x^{2}, 7.9 x^{2}\right), \quad \mathscr{D}_{t_{g H}}^{\frac{3}{4}} v(x, 0)=0 .
\end{array}\right.
$$


We want to find a $1-1-g H$-differentiable solution for this problem. By equation (16) we have

$$
\begin{aligned}
v(x, t) & =\frac{1}{2}\left(f\left(x+\frac{4}{3} t^{\frac{3}{4}}\right) \oplus f\left(x-\frac{4}{3} t^{\frac{3}{4}}\right)\right) \\
& =(1.05,2.5,3.95)\left(\left(x+\frac{4}{3} t^{\frac{3}{4}}\right)^{2}+\left(x-\frac{4}{3} t^{\frac{3}{4}}\right)^{2}\right) .
\end{aligned}
$$

We plot this solution in Figure 2.

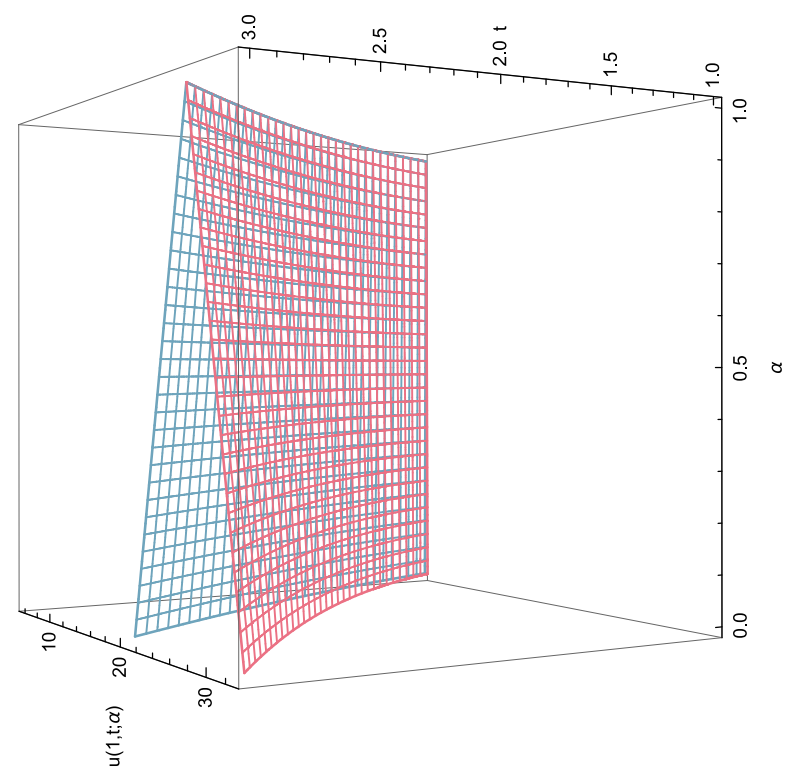

Fig. 2 Representation of $v(x, t)$ for all $r \in[0,1]$ of Example 52

Example 53 Consider the following fuzzy fractional wave equation

$$
\begin{cases}\mathscr{D}_{t t_{g}}^{\frac{14}{8}} v \ominus_{g H} v_{x x_{g H}}=0, & (x, t) \in \mathbb{R} \times(0, \infty), \\ v(x, 0)=\left(2 e^{-x}, 3.6 e^{-x}, 6.5 e^{-x}\right), & \mathscr{D}_{t_{g}}^{\frac{7}{8}} v(x, 0)=0\end{cases}
$$

We want to find a $1-1-g H$-differentiable solution for this problem. By equation (16) we have

$$
\begin{aligned}
v(x, t) & \left.=\frac{1}{2}\left(f\left(x+\frac{8}{7} t^{\frac{7}{8}}\right)\right) \oplus f\left(x-\frac{8}{7} t^{\frac{7}{8}}\right)\right) \\
& =\left(e^{-\frac{8 t^{\frac{7}{8}}}{7}-x}\left(1+e^{\frac{16 t^{\frac{7}{8}}}{7}}\right), 1.8 e^{-\frac{8 t^{\frac{7}{8}}}{7}-x}\left(1+e^{\frac{16 t^{\frac{7}{8}}}{7}}\right), 3.25 e^{-\frac{8 t^{\frac{7}{8}}}{7}-x}\left(1+e^{\frac{16 t^{\frac{7}{8}}}{7}}\right)\right)
\end{aligned}
$$


This solution is plotted in Figure 3.

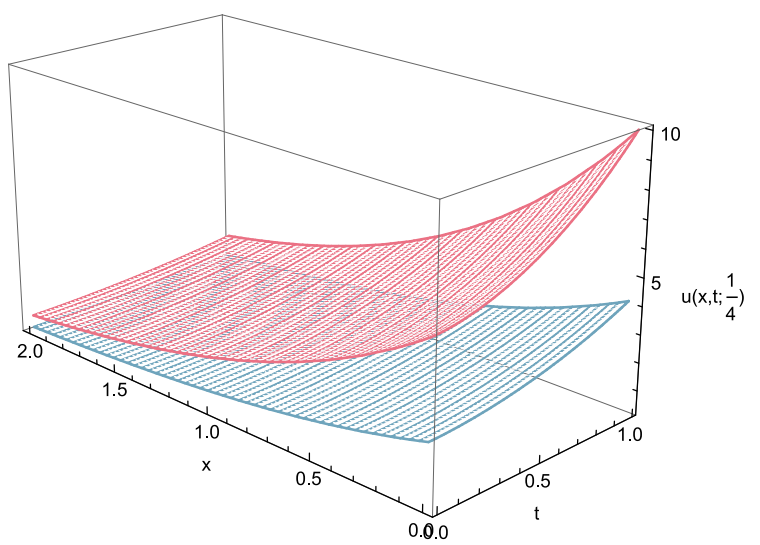

Fig. 3 Graph of $v(x, t)$ of Example 53

\section{Fuzzy Traveling Wave Solution of The Fuzzy Fractional Diffusion Equation}

Consider the following fuzzy fractional linear diffusion equation

$$
\mathscr{D}_{t_{g H}}^{\alpha} v=\mathscr{K} \odot v_{x x_{g H}}
$$

with the initial condition

$$
v(x, 0)=f(x)
$$

where $f(x) \in \mathbb{E}$.

Step 1. To find a traveling wave solution for equation (21), consider

$$
v(x, t)=\mathscr{U}(\xi), \quad \text { where } \xi=x-\mathscr{K} \frac{t^{\alpha}}{\alpha},
$$

where $\mathscr{U}$ is a continuous function and $g H$-differentiable in $\xi$.

Step 2. We have

$$
\frac{\partial \xi}{\partial t}=-\mathscr{K} t^{\alpha-1}<0, \quad \frac{\partial \xi}{\partial x}=1,
$$

Let $v(x, t)$ is $\alpha_{i . g H}$-differentiable with respect to $t$, and $v(x, t)$ and $v_{x_{g H}}$ are $[(i)-$ $p$ ]-differentiable with respect to $x$. Then $\mathscr{U}(\xi)$ is a $[(i)-g H]$-differentiable fuzzy function and

$$
\begin{aligned}
\mathscr{D}_{t_{i . g H}}^{\alpha} v & =t^{1-\alpha} \odot \frac{d_{i . g H} \mathscr{U}}{d \xi} \odot \frac{\partial \xi}{\partial t}=\ominus \mathscr{K} \odot \frac{d_{i . g H} \mathscr{U}}{d \xi} . \\
v_{x_{i . g H}} & =\frac{d_{i . g H} \mathscr{U}}{d \xi} \odot \frac{\partial \xi}{\partial x}=\frac{d_{i . g H} \mathscr{U}}{d \xi}, \Rightarrow v_{x x_{i . g H}}=\frac{d_{i . g H}^{2} \mathscr{U}}{d \xi^{2}} .
\end{aligned}
$$


Hence the equation (21) is reduced to the following fuzzy ordinary differential equation of $\xi$

$$
\frac{d_{i . g H}^{2} \mathscr{U}}{d \xi^{2}} \oplus \frac{d_{i . g H} \mathscr{U}}{d \xi}=0 .
$$

Step 3. To find fuzzy solutions for ordinary differential equation (23), we need some auxiliary boundary conditions. Which in this article, we consider the following auxiliary boundary conditions

$$
\lim _{\xi \rightarrow \pm \infty} \mathscr{U}(\xi)=0, \quad \lim _{\xi \rightarrow \pm \infty} \frac{d \mathscr{U}}{d \xi}=0, \quad \lim _{\xi \rightarrow \pm \infty} \frac{d^{2} \mathscr{U}}{d \xi^{2}}=0 .
$$

We integrate both sides of $\mathrm{Eq}(23)$. According to the auxiliary boundary conditions expressed in Eq.(24), the integration constants are zero and

$$
\frac{d_{i . g H} \mathscr{U}}{d \xi} \oplus \mathscr{U}=0
$$

This equation has the following fuzzy solution [4]

$$
U(\xi)=\mathscr{C} e^{-\xi}
$$

which satisfies the condition $U(\xi)=0$ when $\xi \rightarrow \infty$. Therefore

$$
v(x, t)=\mathscr{C} e^{-\left(x-\mathscr{K} \frac{t^{\alpha}}{\alpha}\right)} .
$$

Using the initial condition (22), we can write

$$
\mathscr{C}=f(x) e^{x}
$$

and finally the fuzzy solution for the fuzzy linear diffusion equation is equal

$$
v(x, t)=f(x) e^{\mathscr{K} \frac{t^{\alpha}}{\alpha}} .
$$

The other case of differentiability can be examined in a similar way.

Example 61 Consider the following fuzzy fractional Diffusion equation

$$
\left\{\begin{array}{l}
\mathscr{D}_{t_{g H}}^{\frac{1}{3}} v=v_{x x_{g H}}, \\
v(x, 0)=\left(3 e^{x}, 6.2 e^{x}, 9.9 e^{x}\right) .
\end{array}\right.
$$

So using equation (26) we have

$$
v(x, t)=\left(3 e^{-x+3 t^{\frac{1}{3}}}, 6.2 e^{-x+3 t^{\frac{1}{3}}}, 9.9 e^{-x+3 t^{\frac{1}{3}}}\right) .
$$

We plot this solution in Figure 4. 


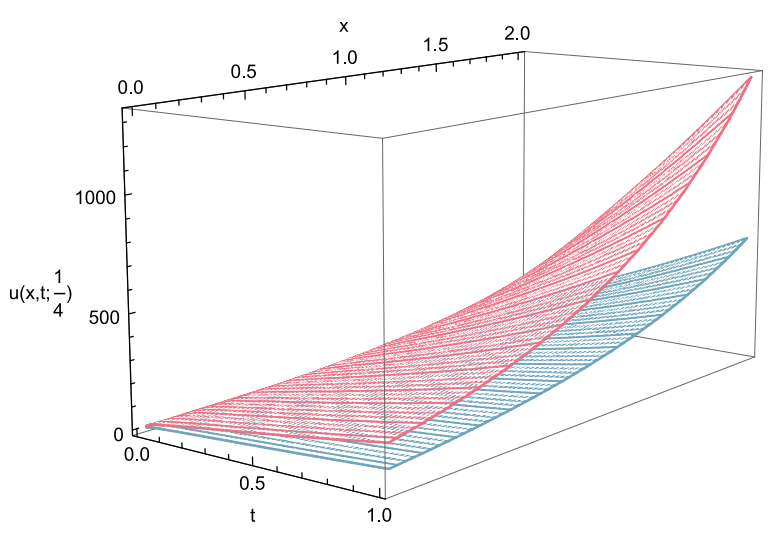

Fig. 4 Graph of $v(x, t)$ for $r=\frac{1}{4}$ of Example 61

\section{Conclusion}

In this paper, we have defined the generalized Hukuhara conformable fractional derivative and the type of differentiability of this derivative is studied in detail, and we have proved some novel properties for it. The fuzzy traveling wave solution of the fractional Wave equation and fractional Diffusion equation was obtained by considering the type of $\alpha_{g H}$-differentiability. To demonstrate the efficiency of the method, the fuzzy traveling wave solutions of some examples were obtained. All results show that this method is a compelling and efficient method for obtaining an analytical solution for the fuzzy linear fractional partial differential equation.

Acknowledgements The authors wish to thank the anonymous referees for their comments and criticism. All of their comments were taken into account in the revised version of the paper, resulting in a substantial improvement with respect to the original submission.

\section{Compliance with Ethical Standards}

\section{Conflict of interest}

The authors declare that they have no conflict of interest.

Ethical approval

This article does not contain any studies with human participants or animals performed by any of the authors. 


\section{References}

1. Allahviranloo, T. (2021) Fuzzy Fractional Differential Equations. In: Fuzzy Fractional Differential Operators and Equations. Studies in Fuzziness and Soft Computing, 397, Springer, Cham

2. Allahviranloo, T., Gouyandeh, Z., Armand, A., Hasanoglu, A. (2015) On fuzzy solutions for heat equation based on generalized hukuhara differentiability, Fuzzy Sets and Systems,265,1-23.

3. Armand, A., Allahviranloo, T., Gouyandeh,Z. (2018) some fundamental results on fuzzy calculus, Iranian Journal of Fuzzy Systems, 15(3), 27-46.

4. Armand, A., Gouyandeh, Z. (2017) On Fuzzy Solution for Exact Second Order Fuzzy Differential Equation,International Journal of Industrial Mathematics, 9, 279-288

5. Agarwal, R.P., Arshad, S., ÓRegan, D., Lupulescu, V. (2012) Fuzzy fractional integral equations under compactness type condition, Fract. Calc. Appl. Anal, 15, 572-590.

6. Arqub, O. A., Al-Smadi, M. (2020) Fuzzy conformable fractional differential equations: novel extended approach and new numerical solutions, Soft Comput, 24, 12501-12522.

7. Arshad, S., Lupulescu, V. (2011) On the fractional differential equations with uncertainty, Nonlinear Anal. 75, 3685-3693.

8. Anastassiou, G. A. (2010) Fuzzy Mathematics: Approximation Theory, Studies in Fuzziness and Soft Computing, Springer-Verlag Berlin Heidelberg.

9. Bede, B. , Gal, S.G. (2005) Generalizations of the differentiability of fuzzy-number-valued functions with applications to fuzzy differential equations, Fuzzy Set and Systems, 151, 581-599.

10. Bede, B., Gal, S.G. (2006) Remark on the new solutions of fuzzy differential equations, Chaos Solitons Fractals.

11. Bede, B. (2013) Mathematics of fuzzy sets and fuzzy logic, Springer, London.

12. Bede, B., Stefanini, L. (2011) Solution of Fuzzy Differential Equations with generalized differentiability using LU-parametric representation, EUSFLAT, 1 , 785-790.

13. Chalco-Cano, Y., Roman-Flores, H., Jimenez-Gamero, M.D. (2011) Generalized derivative and $\pi$ derivative for set-valued functions, Information Sciences, 181, 2177-2188.

14. Chalco-Cano, Y., Costab, T.M., Román-Floresc, H., Rufián-Lizana, A. (2020) New properties of the switching points for the generalized Hukuhara differentiability and some results on calculus, Fuzzy Sets and Systems,https://doi.org/10.1016/j.fss.2020.06.016

15. Chang, S., Zadeh, L. (1972) On fuzzy mapping and control, IEEE Trans Syst Cybern, 2, 30-34.

16. Diamond, P. (2002)Brief note on the variation of constants formula for fuzzy differential equations, Fuzzy Sets Syst, 129, 65-71.

17. Ghobaei-Arani, M., Khorsand, R., Ramezanpour, M. (2019) An autonomous resource provisioning framework for massively multiplayer online games in cloud environment, Journal of Network and Computer Applications, 142, 76-97.

18. Ghobaei-Arani, M., Souri, A.(2019) LP-WSC: a linear programming approach for web service composition in geographically distributed cloud environments. J Supercomput 75, 2603-2628, https://doi.org/10.1007/s11227-018-2656-3

19. Ghobaei-Arani, M., Rezaei, M., Souri, A. (2021)An auto-scaling mechanism for cloudbased multimedia storage systems: a fuzzy-based elastic controller. Multimed Tools Appl . https://doi.org/10.1007/s11042-021-11021-9.

20. Hoa, N. V., Vu, H., Duc, T. M. (2019) Fuzzy fractional differential equations under CaputoKatugampola fractional derivative approach, Fuzzy Sets and Systems, 375, 70-99.

21. Hukuhara, M. (1967) Integration des applications mesurables dont la valeur est un compact convex, Funkcial Ekvac, 10, 205-229.

22. Harir, A., Melliani, S., Saadia Chadli, L.(2020) Fuzzy Generalized Conformable Fractional Derivative, Advances in Fuzzy Systems,https://doi.org/10.1155/2020/1954975.

23. Harir, A., Melliani, S., Saadia Chadli, L.(2021) Fuzzy Conformable Fractional Differential Equations, International Journal of Differential Equations, https://doi.org/10.1155/2021/6655450.

24. Kaleva, O. (1987) Fuzzy differential equations, Fuzzy Sets and Systems, 24, 301-317.

25. Khalil, R., Al Horani, M., Yousef, A., Sababheh, M. (2014) A new definition of fractional derivative, Journal of Computational and Applied Mathematics, 264, 65-70.

26. Khorsand, R, Ghobaei-Arani, M, Ramezanpour, M.(2018)FAHP approach for autonomic resource provisioning of multitier applications in cloud computing environments. Softw Pract Exper, 48, 2147 2173. https://doi.org/10.1002/spe. 2627

27. Mazandarani, M., Xiu, L.,(2021) A Review on Fuzzy Differential Equations," in IEEE Access, 9, 62195-62211, https://doi.org/10.1109/ACCESS.2021.3074245. 
28. Moghaddam, R. G., Allahviranloo, T. (2018) On the fuzzy Poisson equation, Fuzzy Sets Syst., 347, $105-128$

29. Martynyuk, A. A., Stamov, G. T., Stamova, I. M.,(2020) Fractional-like Hukuhara derivatives in the theory of set-valued differential equations, Chaos, Solitons \& Fractals, 131, 109487.

30. Puri, M.L.,Ralescu, D.A. (1986) Differentials of fuzzy functions, J Math Anal Appl, 114, 409-422.

31. Shaha, K., Seadawy, A. R., Arfana, M. (2020) Evaluation of one dimensional fuzzy fractional partial differential equations, Alexandria Engineering Journal, In Press, https://doi.org/10.1016/j.aej.2020.05.003

32. Shahidinejad, A., Ghobaei-Arani, M., Esmaeili, L. (2020) An elastic controller using Colored Petri Nets in cloud computing environment. Cluster Comput 23, 10451071 . https://doi.org/10.1007/s10586019-02972-8.

33. Shahidinejad, A, Ghobaei-Arani, M.(2020) Joint computation offloading and resource provisioning for edge-cloud computing environment: A machine learning-based approach. Softw: Pract Exper., 50, 2212-2230. https://doi.org/10.1002/spe.2888

34. Shahsavari, Z., Allahviranloo, T., Abbasbandy, S., Rostamy-Malkhalifeh, M. (2020). The Traveling Wave Solution of the Fuzzy Linear Partial Differential Equation, Applications and Applied Mathematics: An International Journal, 15(1), Article 23.

35. Senol, M., Atpinar, S., Zararsiz, Z., Salahshour, S., Ahmadian, A. (2019) Approximate solution of time-fractional fuzzy partial differential equations. Comp. Appl. Math. 38. https://doi.org/10.1007/s40314-019-0796-6.

36. Stefanini, L., Bede, B. (2009) Generalized Hukuhara differentiability of interval-valued functions and interval differential equations, Nonlinear Analysis, 71, 1311-1328.

37. Viet Long, H., Thi Kim Son, N. , Thi Thanh Tam, H. (2017) The solvability of fuzzy fractional partial differential equations under Caputo gH-differentiability, Fuzzy Sets and Systems, 309, 35-63

38. Zureigat, H., Ismail, A.I., Sathasivam, S. (2020) A compact Crank-Nicholson scheme for the numerical solution of fuzzy time fractional diffusion equations. Neural Comput \& Applic, 32, 6405-6412. https://doi.org/10.1007/s00521-019-04148-2. 